\title{
Vascular endothelial growth factor expression in dogs suffering from squamous cell carcinoma
}

\author{
ALEKSANDRA SOBCZYŃSKA-RAK, IZABELA POLKOWSKA, MAGDALENA GOŁYŃSKA, \\ TOMASZ SZPONDER, BEATA ŻYLIŃSKA, WOJCIECH ŁOPUSZYŃSKI*
}

\begin{abstract}
Department and Clinic of Animal Surgery, *Department of Pathological Anatomy, Faculty of Veterinary Medicine, University of Life Sciences in Lublin, ul. Głęboka 30, 20-612 Lublin, Poland
\end{abstract}

\section{Sobczyńska-Rak A., Polkowska I., Gołyńska M., Szponder T., Żylińska B., Łopuszyński W. Vascular endothelial growth factor expression in dogs suffering from squamous cell carcinoma}

\section{Summary}

The aim of the present study was to monitor VEGF expression in the blood serum of dogs suffering from squamous cell carcinoma in correlation with the speed of metastasis. The research material consisted of tumour tissue and blood samples collected from 26 dogs of various breeds, aged between 5 and 15 years, and 10 control group animals. Blood samples were centrifuged, and the serum obtained was examined by immunoenzymatic assays to determine vascular endothelial growth factor (VEGF) levels. The study group animals were observed for a period of 6 months, during which their blood was drawn twice for immunoenzymatic tests. In all study group animals, elevated blood serum VEGF levels were observed on the day of surgery compared with the control group. In 14 cases, disease relapse was observed with metastases to the bones or lungs. In all relapsing animals, significantly higher blood serum VEGF levels were observed.

VEGF levels are closely related to the neoplastic process, but the exact mechanism of the factor's involvement in neoplastic progression remains unclear, as does the complexity of the angiogenic processes. Studies of the mechanisms affecting tumour proliferation and the monitoring of VEGF levels can facilitate better diagnostics and prognostics, as well as improve the effectiveness of cancer treatment and the planning of new therapeutic strategies for both human and animal patients.

Keywords: VEGF, squamous cell carcinoma, dogs

Squamous cell carcinoma (carcinoma spinocellulare, carcinoma planoepitheliale, spinalioma, SCC) is a malignant tumour originating from keratinized epidermal cells or non-cornified stratified epithelium. Its clinical picture can be varied and depends on the location of the lesions. It is characterised by quick growth and tendency to metastasise to surrounding tissues (26). Growths can originate from the skin and subcutaneous tissues as well as epithelial cells: in the oral cavity, stomach, lungs, vagina and bladder.

The growth of the original tumour as well as the occurrence of metastasis is largely dependent on neoangiogenesis, i.e. the formation of blood vessels in tumour tissue and the so-called angiogenic phenotype of the tumour cells (18). As a result of angiogenic switch, permanent genetic modifications occur, which lead to uncontrolled production of pro-angiogenic factors, i.e. VEGF FGF, IL-6, and IL-8, inducing neovascularisation in the tumour $(2,6,23)$.

VEGF (vascular endothelial growth factor) plays a key part in tumour progression. It is synthesised by numerous cells, including endothelium, macrophages, T lymphocytes, smooth muscles, keratocytes, fibroblasts, and tumour cells $(2,5,21,23)$. Its expression is largely conditioned by not only tumour cells but also the extracellular matrix and tumour microenvironment cells (6). It is a cytokine with a documented angiogenic effect on the activity of vascular endothelium cells (20). The most important factor inducing the expression of VEGF is hypoxia, due to which HIF 1 alpha protein (Hypoxia Inducible Factor) is expressed, facilitating the adaptation of tumour cells to the lower oxygen concentrations in their environment $(17,20)$. HIF 1 alpha protein intensifies the synthesis and expression of VEGF, which binds with VEGF receptors on the surface of endothelium cells and activates intracellular transmission tracts. This leads to activation of kinases and phospholipases, initiation of endothelial cells' migration, proliferation and increased blood vessel permeability (4). It has been confirmed that apart from its vascular role, VEGF also activates enzymes responsible for the destruction of the extracellular 
matrix and influences the migration of macrophages and formation of Bcl-2 protein, which protects cells against apoptosis $(5,18)$. The vascular growth factor also shows autocrynic qualities, since it functions as a factor facilitating the survival of tumour cells by protecting them against hypoxia, as well as chemotherapy- and radiotherapy-induced stress (5).

Many publications have been published to date discussing the role of angiogenesis in various tumours in humans and animals alike. It has been demonstrated that the number of newly formed blood vessels impacts the development and progression of the disease (20). Furthermore, effective blood supply facilitates the penetration of tumour cells into the blood stream and initiation of metastasis (2).

The aim of the present study was to monitor VEGF expression in the blood serum of the animals studied and to correlate it with their general health and the speed of metastasis.

\section{Material and methods}

The study was performed on 160 oncology patients treated at the Department and Clinic of Veterinary Surgery at the University of Life Sciences in Lublin, Poland. The dogs, aged between 5 and 15 years, were of various breeds. In all animals, prior to the surgical removal of the tumour, general chest $\mathrm{x}$-rays were performed to exclude the presence of metastasis to the lungs.

Tissue material obtained during surgery was submitted for tests to the Pathoanatomy Unit at the Department of Veterinary Medicine at the University of Life Sciences in Lublin. Tumour specimens were preserved for 24 hours in $10 \%$ formalin, $\mathrm{pH}=7.2$, and then progressed through increasing concentrations of alcohol solutions, acetone and xylene to paraffin blocks in a tissue processor (Leica TP-20). Preparations intended for histopathological analysis were stained with hematoxylin and eosin, and subsequently analysed under a light microscope (Nikon Eclipse E-600). Microscopic evaluation of specimens were conducted in accordance with the World Health Organization (WHO) histopatological classification of skin tumours (8).

On the day of surgery, blood was drawn from the animals to obtain blood serum for immunoenzymatic assays. cell carcinoma
After obtaining the results of histopathological tests from the initial group of 160 animals, 26 dogs diagnosed with squamous cell carcinoma were selected. The resected lesions included 11 tumours on the skin of the trunk, 6 in the digit region, and 9 in the oral cavity.

The group of animals selected for the study were observed for a period of 6 months, during which blood was drawn twice for immunoenzymatic tests (one and six months after tumour resection). Control radiological examinations of the lungs or bones and/or abdominal cavity ultrasounds were performed.

The control group comprised 10 dogs aged 2 to 7 years, in general good health, brought in for sterilization.

The owners consented to the participation of the dogs in both the control group and the study group.

Tab. 1. Results of the immunoenzymatic test in the group of dogs with squamous

\begin{tabular}{|c|c|c|c|c|c|}
\hline \multirow[b]{2}{*}{ Breed, sex, age in years } & \multirow{2}{*}{$\begin{array}{l}\text { Time point } 0 \\
\text { VEGF (pg/ml) }\end{array}$} & \multicolumn{2}{|c|}{ Time point 1} & \multicolumn{2}{|c|}{ Time point 2} \\
\hline & & VEGF (pg/ml) & $\begin{array}{l}\text { Tumor } \\
\text { recurrence }\end{array}$ & $\operatorname{VEGF}(p g / m l)$ & $\begin{array}{l}\text { Tumor } \\
\text { recurrence }\end{array}$ \\
\hline \multicolumn{6}{|c|}{ SCC in the oral cavity } \\
\hline Mixed breed, $\partial, 10$ & 63.80 & 74.86 & + & 87.51 & \\
\hline Mixed breed,,, 11 & 24.12 & 46.22 & + & 64.68 & \\
\hline Collie rough, $\partial, 12$ & 29.08 & 18.47 & & 12.02 & \\
\hline German shepherd, $\hat{\partial}, 10$ & 65.21 & 119.28 & + & 307.18 & \\
\hline Cocker spaniel,,, 9 & 26.52 & 42.28 & + & 68.59 & \\
\hline Dachshund, $\curvearrowright, 10$ & 21.18 & 17.50 & & 11.08 & \\
\hline German shepherd, $\widehat{\partial}, 6$ & 46.62 & 52.76 & + & 58.77 & \\
\hline German mastiff, $\widehat{\jmath}, 8$ & 99.19 & 128.64 & + & 166.10 & \\
\hline Mixed breed, $\partial^{\lambda}, 7$ & 26.82 & 48.40 & + & 65.43 & \\
\hline \multicolumn{6}{|c|}{ SCC in the digit region } \\
\hline Bulmastiff,,, 9 & 28.30 & 24.20 & & 164.80 & + \\
\hline Rottweiler, $\curvearrowright, 10$ & 107.18 & 86.92 & & 128.41 & + \\
\hline Mixed breed, $\hat{\partial}, 11$ & 65.28 & 22.21 & & 14.13 & \\
\hline Labrador retriever, $\widehat{\partial}, 9$ & 87.51 & 42.69 & & 99.19 & + \\
\hline Schnauzer Giant, $\widehat{\jmath}, 8$ & 65.21 & 42.76 & & 18.59 & \\
\hline Boxer,, , 8 & 34.66 & 89.82 & & 307.18 & + \\
\hline \multicolumn{6}{|c|}{ SCC on the skin of the trunk } \\
\hline German shepherd,,+12 & 52.55 & 24.30 & & 13.09 & \\
\hline Mixed breed, $\uparrow, 9$ & 164.10 & 29.87 & & 84.77 & + \\
\hline Poodle, đ̂, 14 & 35.40 & 32.21 & & 21.33 & \\
\hline Yorkshire terrier, ${ }^{7}, 6$ & 28.30 & 13.42 & & 11.08 & \\
\hline Mixed breed,,, 10 & 24.62 & 22.63 & & 19.87 & \\
\hline Mixed breed, $\widehat{\jmath}, 6$ & 80.11 & 20.19 & & 128.19 & + \\
\hline Boxer, §̊, 5 & 68.29 & 32.63 & & 88.36 & + \\
\hline Maltese,,+6 & 54.82 & 13.21 & & 11.09 & \\
\hline Mixed breed,,, 11 & 64.10 & 22.89 & & 14.82 & \\
\hline German shepherd,,+9 & 64.22 & 27.40 & & 26.20 & \\
\hline Boxer, $\overparen{\partial}, 7$ & 79.46 & 24.69 & & 21.18 & \\
\hline
\end{tabular}

Explanations: time point 0 : on the day of surgery; 1: one month after surgery; 2: six months after surgery; $(+)$ : metastases 
The blood samples were separated by centrifugation, and the vascular endothelial growth factor (VEGF) content was determined in blood serum. The tests were performed with the ELISA immunoenzymatic assay (Quantikine Canine Immunoassay; R\&D Systems, INC Minneapolis, USA, supplier BIOKOM). All stages of the determination were performed in accordance with the vendor's recommendations.

Statistical analysis was carried out to verify any significant correlations between the level of VEGF in the serum of sick animals and of the control group, and to determine whether VEGF values may prove useful in monitoring the health of an animal in the post-surgery period of at least six months. The Mann-Whitney test was used to verify the significance of discrepancies observed between the two groups when the variables were not normally distributed.

\section{Results and discussion}

Patient characteristics and blood serum VEGF levels in the study group animals are presented in Tab. 1.

On the day of the surgery, elevated blood serum VEGF levels were observed in all study group animals, ranging from 21.18 to $307.18 \mathrm{pg} / \mathrm{ml}$ (compared to the control group levels of $0.1-13.04 \mathrm{pg} / \mathrm{ml})$. The result was statistically significant $(\mathrm{P}<0.0001)-$ Fig. 1 . In fourteen dogs under observation, disease relapse occurred with metastases to the bones or lungs. In seven dogs suffering from oral cavity tumours, the remission period lasted only one month. In two cases, the relapse occurred in the oral cavity soft tissue; in three animals metastases to the jaw or mandible bone were observed; and in the remaining two dogs metastases to lung tissue were diagnosed.

The blood serum VEGF levels were higher than on the day of the surgery, although the results were not statistically significant $(\mathrm{P}>0.05)$. Statistical significance for blood serum levels in the cases of oral cavity SCC was observed only after 6 months $(\mathrm{P}<0.05)-$ Fig. 2 . In seven dogs, metastases were observed six months after the surgery. In four animals initially diagnosed with tumours in the digit region, metastases were found in long limb bones $(\mathrm{P}<0.01)$. The remaining three cases were metastases to the lungs in animals initially diagnosed with trunk skin SCC.

In all relapsing animals, significantly higher blood serum VEGF levels were observed, which oscillated between 46.28 and $307.18 \mathrm{pg} / \mathrm{ml}$ (median $=93.77 \mathrm{pg}$ / $\mathrm{ml})$. In comparison to the VEGF levels observed on the day of surgery, the results were statistically significant $(\mathrm{P}<0.01)-$ Fig. 3.

In dogs suffering from trunk skin SCC that showed signs of remission after tumour resection, blood serum VEGF levels were significantly lower $(\mathrm{P}<0.05)$ Fig. 4.

Numerous locations, macroscopic pictures of the lesions, and symptoms related to the presence of squamous cell carcinoma have already been discussed. Despite considerable variability of its location, the macroscopic characteristics of squamous cell carci-

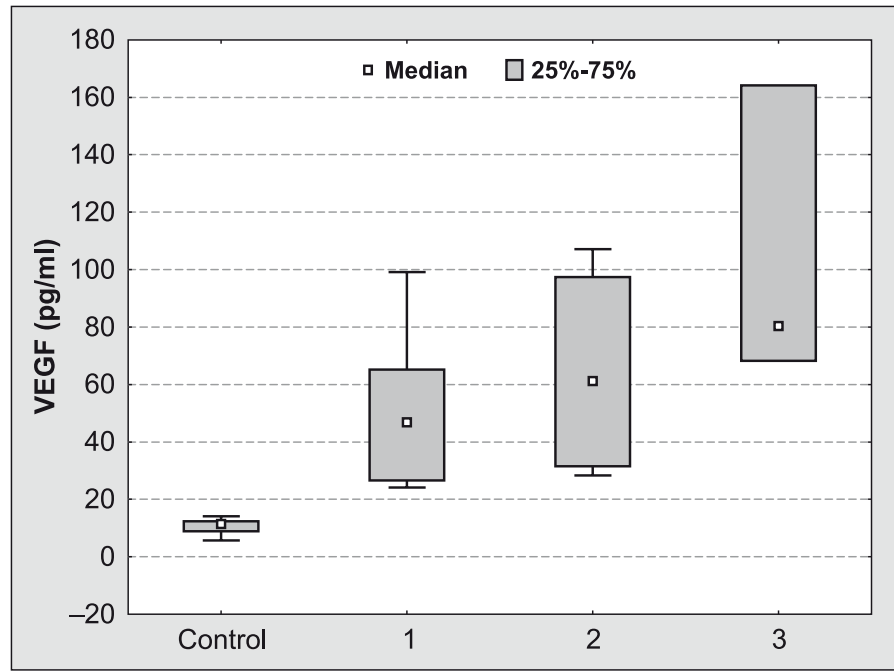

Fig. 1. VEGF in the blood serum of dogs suffering from squamous cell carcinoma compared to the control group

Explanations: Time point: 1 - SCC in the oral cavity on the day of surgery; 2 - SCC in the digit region on the day of surgery; 3 - SCC on the skin of the trunk on the day of surgery

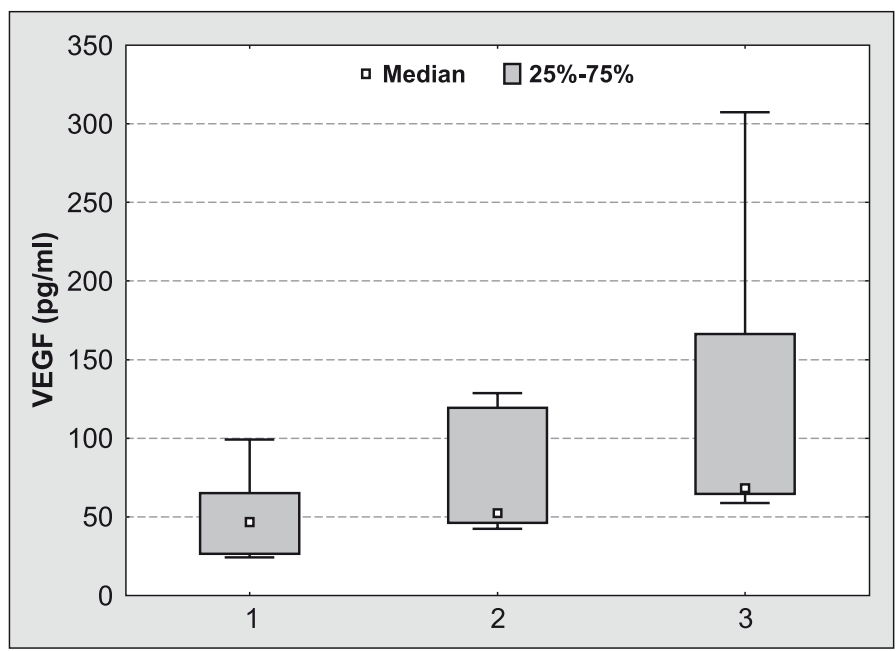

Fig. 2. Blood serum VEGF levels in dogs diagnosed with tumour relapse (metastases) in cases of oral SCC

Explanations: Time point: 1 - on the day of surgery; 2 - one month after surgery; 3 - six months after surgery

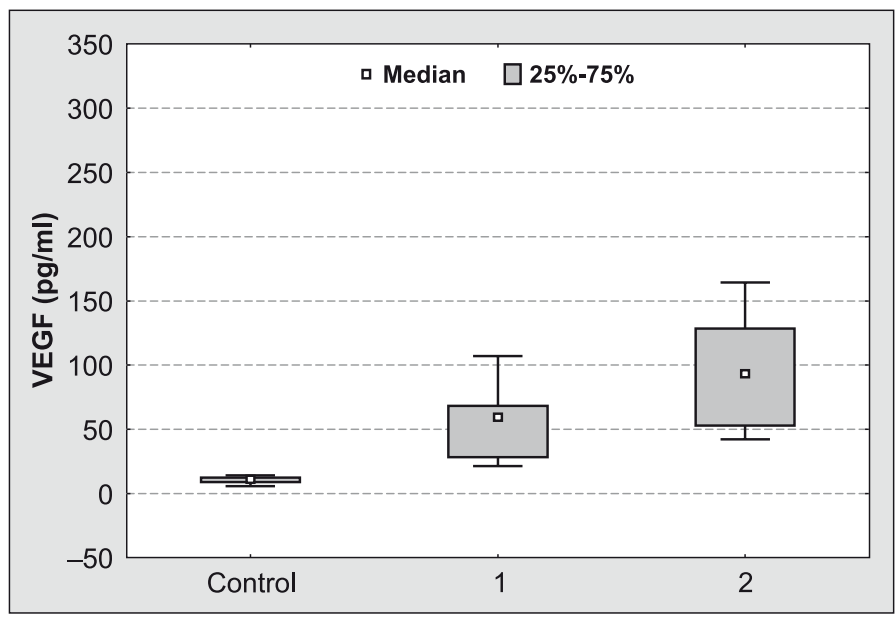

Fig. 3. VEGF levels in the blood serum of metastatic dogs compared to the control group

Explanations: Time point: 1 - on the day of surgery; 2 - one month after surgery 


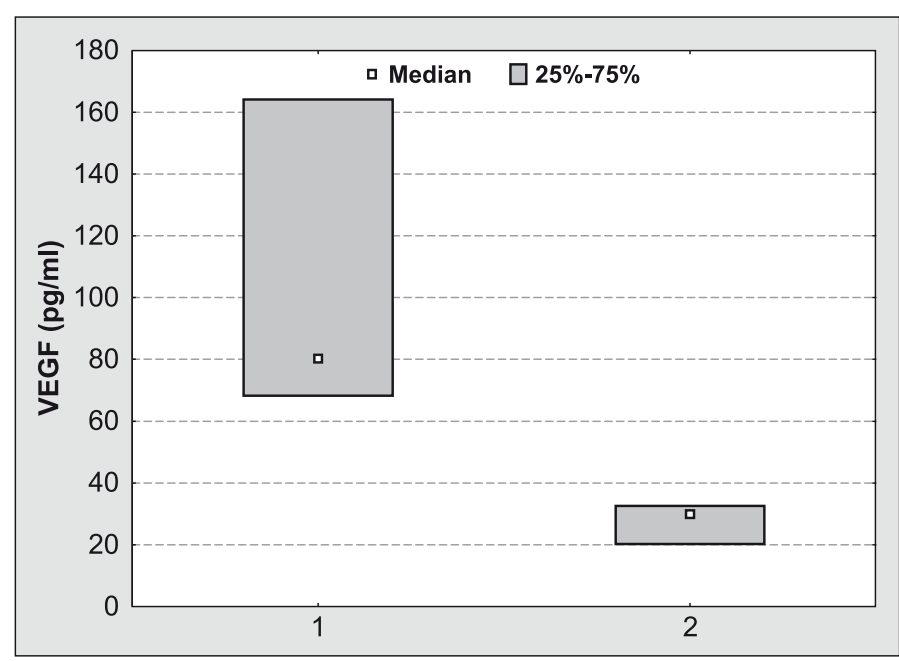

Fig. 4. VEGF levels in the blood serum of dogs suffering from trunk skin SCC in the period of remission

Explanations: Time point: 1 - on the day of surgery; 2 - one month after surgery

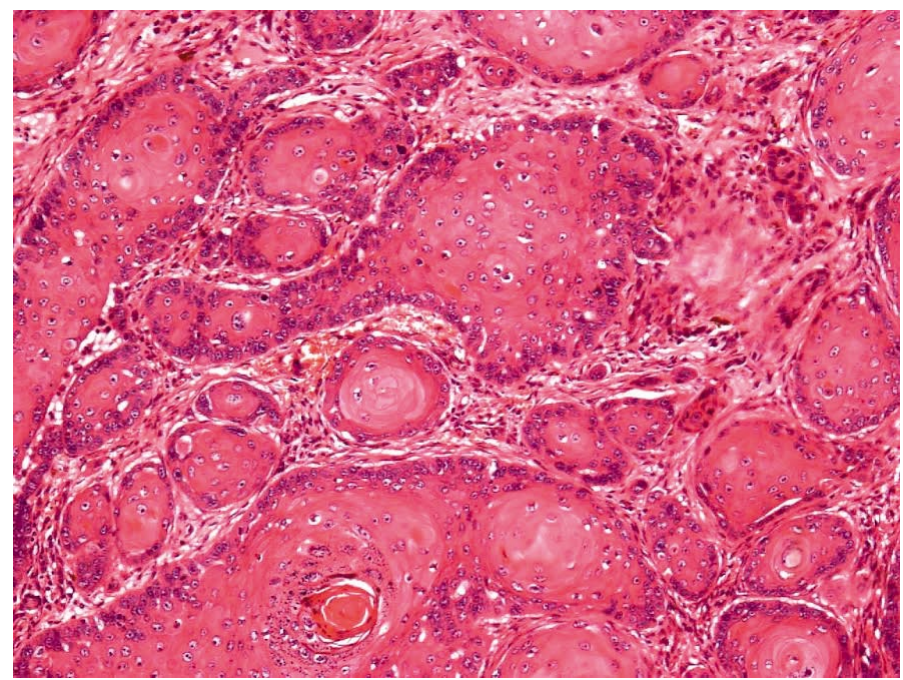

Fig. 5. Histopathology: Squamous cell carcinoma - keratin pearls. Original magnifications: $200 \times$; hematoxylin-eosin staining

noma reveal a number of common features. Cancer cells display the ability to produce keratin and deposit it extracellularly in the form of so-called keratin pearls (Fig. 5). Keratin is treated as a foreign body and causes a strong irritant reaction, which results in inflammation in the region of the lesion (9).

Tumour cell morphology depends on the degree of tumour differentiation, which affects the natural course of the disease and the effectiveness of treatment. Cancers characterised by high or medium differentiation typically progress more slowly (usually locally), and metastases are observed mainly in the lymph nodes. Metastasis to distant organs is relatively rare (10-20\% of cases). Cancers with low differentiation and non-differentiated tumours are characterised by faster local growth (high rate of local recurrence), show a tendency for early metastasis to the lymph nodes and much more commonly metastasise to distant organs up to $40 \%(9,10,26)$.
Squamous cell carcinomas constitute approximately $5 \%$ of all skin tumours diagnosed in dogs (26). Lesions are most often observed in scarcely hirsute or non-pigmented skin regions exposed to UV radiation. Tumour cells often proliferate to the dermis and subcutaneous, tissue, and are characterised by local aggressivity, with infrequent distant metastases observed in the late stages of the disease (9). In the case of tumours located in the regions of the head, neck or trunk, no breed or age predispositions have been observed. The only exception is SCC in the digit region, which has been demonstrated to display both breed and age predispositions, being the most common in large breed dogs: Rottweiler, Labrador, Giant Schnauzer or Dachshund, aged 8-10 years $(10,26)$. Initially, the tumour develops in the form of a lesion accompanied by a swelling in the digital or interdigital region. In time, it grows larger and becomes ulcerated. The process can last up to several months and be accompanied by deteriorating symptoms of lameness. The most effective treatment of digital SCC involves radical surgery with resection of the tumour and amputation of the affected digit or digits.

Some authors report that despite radical surgery, in $5.7-24.1 \%$ of cases metastases occur to the humerus bone, femoral bone, or spine, less frequently to the pelvic bone. This fact affects the survival rate of sick dogs, which fluctuates at approximately $57 \%$ survival up to one year after surgery and $36 \%$ survival up to two years $(10,29)$.

The same was confirmed in the present study, in which four out of six animals showed symptoms of bone metastases in the period of six months after a surgical resection of the tumour.

Squamous cell carcinoma is one of the three most common malignant tumour types observed in canine oral cavities. Factors facilitating accelerated development of the lesions include increased exposure to harmful environmental conditions and chronic parodontitis. Lesions are located on the gums, soft and hard palate, tongue, and buccal mucosa (26). Due to their location, lesions often remain unnoticed by the owner until advanced stages of the disease. The tumours often infiltrate oral mucosa and splanchnocranium muscles. In advanced stages, metastasis to cranial bones and local lymph nodes occur, which affects the extent of necessary surgical intervention. In such cases, resection should extend to the maxillary or mandibular bone (maxilectomy or mandibulectomy).

Microscopically, oral SCC belongs to the group of differentiated tumours with a relatively high potential for metastasis, also to the lungs. The same was confirmed in the present study. In the group of dogs analysed, in seven cases $(77.7 \%)$ bone and lung metastases were observed fairly soon - within one month of the radical resection.

Neovascularisation within the tumour tissue is considered a condition of tumour progression. Chronic, 
VEGF-induced activation of endothelial cell division leads to the reconstruction of the existing blood vessels and formation of news ones, which results in their growth and increased permeability. In this way, VEGF, produced in large quantities by the tumour, becomes a factor responsible for penetration of the endothelial barrier, thus allowing tumour cells to infiltrate beyond the vascular bed and form metastases $(5,11,18)$.

Some authors report a positive correlation between the level of VEGF expression and the clinical symptoms of tumour malignancy, such as tumour advancement, metastasis and relapse $(7,24)$.

Elevated VEGF expression, both in terms of mRNA in the tumour tissue and the protein itself in the blood serum or urine, has been reported in various tumours in humans, including colorectal cancer, gastric cancer, breast cancer, pulmonary cancer, prostate cancer, kidney cancer, and bladder cancer $(5,6,11$, 20, 21).

Elevated levels of VEGF in comparison to its levels in the blood serum of healthy animals have also been reported in veterinary oncology. Overexpression of VEGF has been reported in cases of cancer of the skin, oral cavity, lactiferous gland, spleen tumours, as well as in dogs suffering from multicentric lymphoma, hemangiosarcoma and soft tissue sarcomas $(1,3,7$, $10,12,14,15,17,22,24,25,27,28)$.

Troy et al. (25), who compared glycoprotein levels in healthy dogs with those observed in the blood serum of dogs suffering from cancer, reported significantly higher VEGF levels in the sick animals (2.5-274 pg/ $\mathrm{ml}$ ), which reached prognostic values. Other researchers have proved that high levels of VEGF in the blood serum of animals suffering from malignant melanomas were correlated with shorter survival periods $(\mathrm{P}=0.002)$, and overexpression of the growth factor was significantly higher in dogs in advanced stages of the disease (24).

Similar results were reported by Wergin and KaserHotz (27), who observed that the blood serum VEGF level in dogs suffering from oncological conditions was not affected by their age, sex or body mass, but did correlate with particular types of tumours $(\mathrm{P}=0.02)$. The highest VEGF levels were reported in malignant tumours, especially in melanomas and sarcomas, which confirms the close correlation between VEGF and tumour aggressivity.

Other researchers (14) compared VEGF overexpression in tumours with VEGF in a group of healthy animals $(P<0.01)$, and hypothesised that it could constitute a promising biomarker of neoplastic transformation.

De Queiroz et al. (16) observed increased VEGF expression in cases of soft tissue sarcomas in dogs. They reported that VEGF was present not only in tumour cells, but also in the peritumour region. In subsequent studies conducted on a group of $25 \mathrm{dogs}$, they also observed that the blood serum VEGF level in sick animals was reduced after the resection of the sarcoma (15).

In the present study, conducted in a group of dogs suffering from squamous cell carcinoma, similar results were obtained. Expression of VEGF in the blood serum of most animals with a skin tumour located on the trunk decreased already within one month after the surgery. Growth factor levels showed a general downward trend until the end of the observation period (six months). The results obtained confirm Gross's conclusions with regard to the low metastasis potential of squamous skin carcinoma of the skin. This may be related to the early diagnosis of lesions, which can be more easily noticed by the owners compared with e.g. lesions in the oral cavity. Moreover, skin tumours offer the possibility of a precise as well as macroscopically and microscopically radical resection of the lesion.

The incidence and survival rates in cases of oral SCC in humans are the subject of intensive studies conducted worldwide $(12,20,27)$. The presence of VEGF has been reported not only in tumour cells as such, but also in tumour stroma, which significantly impacts the uncontrolled hyperplasia of the tumour and the incidence of metastases (13). Those studies have made it possible to use the expression of VEGF and its receptors as a significant prognostic factor in cases of SCC of the oral cavity in humans as well as SCC of the oral cavity and nasal epithelium in animals. Thus it is possible to monitor the development and local relapse of the disease, as well as the incidence of distant metastases (19).

The present research indicated that the increase in blood VEGF levels in dogs suffering from skin SCC in the digital region and SCC of the oral cavity correlated with the incidence of local relapse or distant metastases to lung or bone tissues $(\mathrm{P}<0.01)$.

The results obtained suggest that VEGF may serve as a diagnostic and prognostic marker in cases of squamous cell carcinoma of the skin and oral cavity in dogs.

VEGF levels are closely related to the neoplastic process, but the exact mechanism of the factor's involvement in neoplastic progression remains unclear, as does the complexity of the angiogenic processes. Studies of the mechanisms affecting tumour proliferation and the monitoring of VEGF levels can facilitate better diagnostics and prognostics, as well as improve the effectiveness of cancer treatment and the planning of new therapeutic strategies for both human and animal patients.

\section{References}

1. Al Dassi A. N., Haines D. M., Singh B., Kidney B. A.: Immunohistochemical expression of Vascular endothelial growth factor and Vascular endothelial growth factor receptor 2 in canine simple mammary gland adenocarcinomas. Can. Vet. J. 2010, 51, 1109-1114.

2. Bałan B. J., Stotwiński R.: VEGF and tumor angiogenesis. Centr. Eur. J. Immunol. 2008, 33, 232-236.

3. Campos A. G., Campos J. A. D. B., Sanches D. S., Dagli M. L. Z., Matera J. M.: Immunohistochemical evaluation of Vascular Endothelial Growth Factor (VEGF) in splenic hemangiomas and hemangiosarcomas in dogs. Open J. Vet. Med. 2012, 2, 191-195. 
4. Dvorak H. F.: Vascular permeability factor/vascular endothelial growth factor: a critical cytokine in tumor angiogenesis and potential target for diagnosis and therapy. J. Clin. Oncol. 2002, 20, 4368-4380.

5.Ferrara N.: Vascular Endothelial Growth Factor as a Target for Anticancer Therapy. Oncologist 2004, 9, 10-17.

6. Flak B., Wawrzyniec K., Kwiatek S., Kawczyk-Krupka A., Czuba Z., SierońStołtny K., Sieroń A.: Vascular endothelial growth factor (VEGF) as a marker for cancer progression - a review. Acta. Bio-Opti. Inf. Med. Biomed. Eng. 2013, 4, 205-209.

7. Frenz M., Kaup F. J., Neumann S.: Serum vascular endothelial growth factor in dogs with haemangiosarcoma and haematoma. Res. Vet. Sci. 2014, 97, 257-262.

8. Goldschmidt M. H., Dunstan R. W., Stannard A. A., von Tscharner C., Walder E. J., Yager J. A.: Histological Classification of Epithelial and Melanocytic Tumors of the Skin of Domestic Animals. WHO International Histological Classification of Tumors of Domestic Animals, second series, vol. III. Armed Forces Institute of Pathology, Washington D.C. 1998.

9. Gross T. L., Ihrke P., Walder E. J., Affolter V. K.: Skin Diseases of the Dog and Cat. Clinical and Histopathologic Diagnosis. Blackwell Science, Ltd Oxford, UK 2005, pp 932-936.

10. Lima T. B., Leal L. M., Morato G. O., Marinho P. V. T., Garrido E., Moraes P. C., De Nardi A. B.: Metastasis of squamous cell carcinoma in the pelvis of a dog. Acta Sci. Vet. 2013, 41 (Suppl 1), 1-5.

11. Łojko A., Komarnicki M.: Vascular endothelial growth factor in tumor angiogenesis. Współcz. Onkol. 2004, 8, 1-4.

12. Maiolino P., De Vico G., Restucci B.: Expression of Vascular Endothelial Growth Factor in Basal Cell Tumours and in Squamous Cell Carcinomas of Canine Skin. J. Comp. Pathol. 2000, 123, 141-145.

13. Mărgăritescu C., Pirici D., Simionescu C., Mogoantă L., Raica M., Stîngă A., Ciurea R., Stepan A., Stîngă A., Ribattin D.: VEGF and VEGFRs expression in oral squamous cell carcinoma. Rom. J. Morphol. Embryol. 2009, 50, 527-564.

14. Mukorera $V$.: Expression of vascular endothelial growth factor in dogs with Spirocerca lupi-associated neoplastic transformation. J. Vet. Intern. Med. 2013, 27, 1642-1645.

15. Queiroz G. F. de, Dagli M. L., Meira S. A., Matera J. M.: Serum vascular endothelial growth factor in dogs with soft tissue sarcomas. Vet. Comp. Oncol. 2013, 11, 230-235.

16. Queiroz G. F. de, Franchini M. L., Garcez Neto A. F., Matera J. M.: Serum and plasma vascular endothelial growth factor in healthy dogs. Braz. J. Vet. Res. Anim. Sci. 2013a, 5, 359-363.

17. Rodigheri S. M., Calazans S. G., Amorim R. L., De Nardi A. B., Sequeira J. L., Daleck C. R.: Serum concentration and immunostaining of vascular endothelial growth factor in dogs with multicentric lymphoma. Semina: Ciências Agrárias, Londrina 2015, 36, 2649-2660.

18. Senger D. R.: Vascular endothelial growth factor: much more than an angiogenesis factor. Mol. Biol. Cell. 2010, 21, 377-379.

19. Shiomitsu K., Johnson C. L., Malarkey D. E., Pruitt A. F., Thrall D. E. Expression of epidermal growth factor receptor and vascular endothelial growth factor in malignant canine epithelial nasal tumours. Vet. Comp. Oncol. 2009, 7, 106-114.

20. Stodkowska J.: The quantitative estimation of angiogenesis in lung carcinoma and its prognostic significance in the present clinical studies. Pneumonol. Alergol. Pol. 2002, 70, 318-325

21. Sobczyńska-Rak A.: The role of VEGF in process of neovasculogenesis, [in:] Ran S. (ed.) Tumor Angiogenesis. Intech, Rijeka, Croatia 2012, p. 181-196.

22. Sobczyńska-Rak A., Brodzki A.: VEGF and 17-beta-estradiol levels after tamoxifen administration in canine hepatoid gland adenomas and hepatoid gland epitheliomas. In Vivo 2014, 28, 871-878.

23. Szala S.: Angiogenesis and immune suppression: yin and yang of tumor progression? Postępy Hig. Med. Dośw. 2009, 63, 598-612.

24. Taylor K. H., Smith A. N., Higginbotham M., Schwartz D. D., Carpenter D. M., Whitley E. M.: Expression of vascular endothelial growth factor in canine oral malignant melanoma. Vet. Comp. Oncol. 2007, 5, 208-218.

25. Troy G. C., Panciera D., Lanz O., Robertson J. L., Ward D. L.: Endostatin and Vascular Endothelial Growth Factor concentrations in healthy dogs, dogs with selected neoplasia, and dogs with nonneoplastic diseases. J. Vet. Intern. Med. 2006, 20, 144-150

26. Webb J. L., Burns R. E., Brown H. M., LeRoy B. E., Kosarek C. E.: Squamous cell carcinoma. Compend. Contin. Educ. Vet. 2009, 31, 133-142.

27. Wergin M. C., Kaser-Hotz B.: Plasma Vascular Endothelial Growth Factor (VEGF) measured in seventy dogs with spontaneously occurring tumours. In Vivo 2004, 18, 15-20.

28. Wergin M. C., Roos M., Inteeworn N., Laluhovà D., Allemann K., KaserHotz B.: The influence of fractionated radiation therapy on plasma vascular endothelial growth factor (VEGF) concentration in dogs with spontaneous tumors and its impact on outcome. Radiother. Oncol. 2006, 2, 239-244.

29. Wobeser B. K., Kidney B. A., Powers B. E., Withrow S. J., Mayer M. N., Spinato M. T., Allen A. L.: Diagnoses and clinical outcomes associated with surgically amputated canine digits submitted to multiple veterinary diagnostic laboratories. Vet. Pathol. 2007, 44, 355-361.

Corresponding author: Aleksandra Sobczyńska-Rak PhD, Department and Clinic of Animal Surgery, Faculty of Veterinary Medicine, University of Life Sciences in Lublin, Głęboka 30, 20-612 Lublin, Poland; e-mail: olsob2@gmail.com 